\title{
Electroexplosive Technology of Nanopowders Production: Current Status and Future Prospects
}

\author{
Young-Soon Kwon, Jin-Chun Kim, Alexander P. Ilyin ${ }^{a}$, \\ Olga B. Nazarenko ${ }^{a} *$, and Dmitry V. Tikhonov ${ }^{a}$ \\ Research Center for Machine Parts and Materials Processing, School of Materials Engineering, \\ University of Ulsan, San-29, Mugeo-2Dong, Nam-Ku, Ulsan 680-749, Korea \\ ${ }^{a}$ Tomsk Polytechnic University, Lenin str. 30, 634050, Tomsk, Russia \\ (Received January 12, 2012; Revised January 26, 2012; Accepted February 5, 2012)
}

\begin{abstract}
The current situation of the nanopowders production technology based on the process of electrical explosion of wires is described. The advantages and disadvantages of the electroexplosive technology are indicated. The results of studies characterizing the effect of the electrical explosion conditions on the nanopowders properties are presented, including latest results: conditions of nanopowders passivation, conditions of nanopowders production having narrow size distribution, the methods of nanopowders diagnostic and standartization. In addition, the application and area of future research on this technology are proposed.
\end{abstract}

Keywords: Nanopowder, Electrical explosion of wires, Particles characterization

\section{Introduction}

The electrical explosion of wires (EEW) is a process of explosive destruction of a metal wire under the action of great density current $\left(>10^{10} \mathrm{~A} / \mathrm{m}^{2}\right)$. This process is accompanied by scattering products, shock waves and electromagnetic radiation. EEW is characterized by the following peculiarities: time of explosion is $10^{-5} \ldots 10^{-8} \mathrm{~s}$; temperature at the moment of explosion can reach the value more than $10^{4} \mathrm{~K}$, pressure $\sim 10^{9} \mathrm{~Pa}$; velocity of product recession is from 1000 to $5000 \mathrm{~m} / \mathrm{s}$. Material of the wire transmutes into the nanoscale particles $(<100 \mathrm{~nm})$ in accordance with certain conditions. Extremely nonequilibrium conditions of EEW cause some unusual properties of nanopowders. They are steady against oxidation and sintering at room temperature and characterized with high diffusion activity at the heating.

The EEW technology allows producing nanopowders of metals, alloys, intermetallic compounds, and chemical compounds depending on working ambient in the discharge chamber. EEW in inert gases or hydrogen is used to produce powders of metals, alloys, and intermetallic compounds. EEW in chemically active ambient is used to produce nanopowders of chemical compounds of metals. The following nanopowders were obtained by EEW: Ag, $\mathrm{Al}, \mathrm{Cu}, \mathrm{Fe}, \mathrm{Ni}, \mathrm{Ti}, \mathrm{W}, \mathrm{Mo}, \mathrm{Fe}-\mathrm{Ni}, \mathrm{Pb}-\mathrm{Sn}, \mathrm{Cu}-\mathrm{Zn}, \mathrm{Ni}-\mathrm{Cr}$, Al-Cu, Al-B, W-Al, Cu-Ni, Ni-Ti, $\mathrm{Al}_{2} \mathrm{O}_{3}, \mathrm{CuO}, \mathrm{AlN}, \mathrm{W}_{2} \mathrm{C}$, $\mathrm{WC}, \mathrm{TiC}$, etc [1-11].

Technology of nanopowders production based on the phenomenon of EEW has been developed intensively in the last 30 years both in Russia, particularly in Tomsk Polytechnic University, and abroad. The most significant results of our studying the process of EEW as a method for metal and chemical compounds nanopowders production is presented in this paper.

\section{Electrical Explosion of Wires General Aspects}

\subsection{Advantages of the electroexplosive technology}

The method of EEW has a lot of advantages compared to others [1-3]:

- The important advantage of the electroexplosive tech-

*Corresponding Author: O. B. Nazarenko, TEL: +7-3822-563784, FAX: +7-3822-563650, E-mail: obnaz@mail.ru 
nology is the possibility of the control over the properties of the EEW products, including particles size, phase and chemical composition of nanopowders by means of electrical parameters.

- This method is characterized with low energy consumption-less than $10 \ldots 20 \mathrm{kWh} / \mathrm{kg}$, due to direct heating of the wire by electric current without additional heating equipments and due to high heating rate $\left(>10^{7} \mathrm{~K} / \mathrm{s}\right)$ that provides adiabatic conditions of energy transmission to the wire.

- The productivity is high enough: the output of nanopowders on the basis of aluminium wires explosion is $50 \mathrm{~g} / \mathrm{h}$, on the basis of tungsten wires explosion $300 \mathrm{~g} / \mathrm{h}$.

- The electroexplosive technology, unlike other technologies, allows using the same installation to produce nanopowders of metals, alloys, intermetallic compounds, and chemical compounds depending on working ambient in the discharge chamber.

- The electroexplosive technology is environmentally benign one: the process of nanopowder production is carried out in closed chamber, there is no technological emission.

\subsection{The process of EEW}

Electrical explosion of wires is a process of explosive destruction of a metal wire under the action of great current density $\left(>10^{10} \mathrm{~A} / \mathrm{m}^{2}\right)$. This process is accompanied by scattering products, shock waves and electromagnetic radiation. Material of the wire transmutes into the particles of nanosized range in accordance with certain conditions. EEW is characterized by the following peculiarities: time of explosion is $10^{-5} \ldots 10^{-8} \mathrm{~s}$; temperature at the moment of explosion is more than $10^{4} \mathrm{~K}$, pressure $\sim 10^{9}$ $\mathrm{Pa}$; velocity of product recession is $1 \ldots .5 \mathrm{~km} / \mathrm{s}$.

The process of EEW includes the following stages: heating of metal in solid state, melting, heating of fluid metal before beginning vaporization, expansion of wire material, scattering of primary products and formation of the final particles.

\subsection{Nonequilibrium state of EEW products}

The formation of nanosized particles in EEW process occurs in the presence of strongly non equilibrium conditions - the large gradients of temperature and speeds of

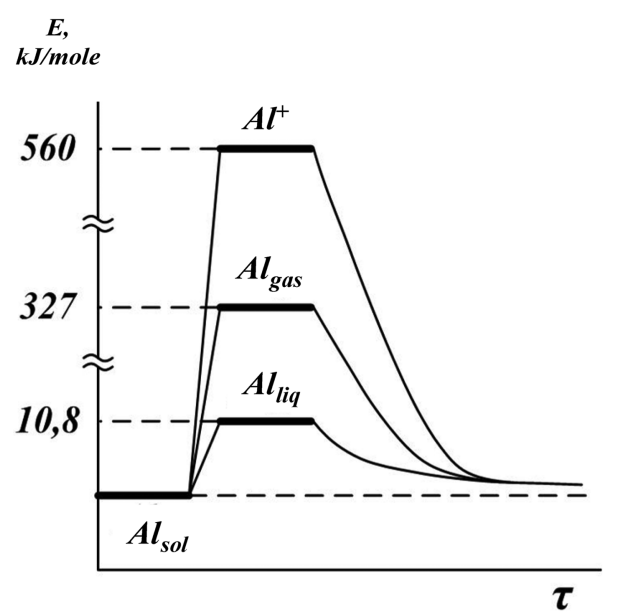

Fig. 1. The energy intensity of processes at the action of concentrated energy flow on aluminum.

cooling $\left(>10^{7} \mathrm{~K} / \mathrm{s}\right)$, intensive heat transfer and mass transfer, ionization, mixture, influence of electric and magnetic fields. The extreme conditions of EEW process provide thermodynamically nonequilibrium structure of metal nanopowders and at the same time it is enough steady structure and presence of the excess stored energy $[12,13]$.

During the fast explosion a metal wire proceeds to a non-equilibrium state which is characterized by two types of non-equilibrium: the phase and temperature non-equilibrium state. The thermal nonequilibrium is that the result of passing an electric current through a metal wire and its destruction an excitation of the electronic subsystem occurs (to a temperature of $10^{6} \mathrm{~K}$ ), but the atomic subsystem is colder $\left(10^{4} \mathrm{~K}\right)$. The phase nonequilibrium is that the primary products are vapor (clusters), plasma and overheated liquid drops, which cannot coexist under equilibrium conditions (Fig. 1).

\section{The Methods for Regulation of EEW-nanopowders Characteristics}

Nanoparticles produced by EEW have spherical shape; at the same time nanopowders are polydisperse systems $[1,3]$. The range of particles size of the electroexplosive nanopowders is rather wide: from $5 \cdot 10^{-9}$ to $10^{-3} \mathrm{~m}$. Powder dispersiveness is one of the most important parameters that determines their technical characteristics (packed density, flow rate, slope angle, reaction capacity and others), and their range of use. At the decreasing of metal 
particles size less than $100 \mathrm{~nm}$ their reactivity increases but the metal content in particles decreases simultaneously. For instance, the content of metal aluminum in products of electrical explosion of aluminum wires in the medium of hydrogen is $93.4 \%$ for nanopowder with average surface particle diameter $290 \mathrm{~nm}$ and $86.3 \%$ for nanopowder with average surface particle diameter $120 \mathrm{~nm}$. Another problem of the nano-state is the nanopowder agglomeration caused the active particle surfaces. Nanopowders with particle sizes less than $30 \mathrm{~nm}$ are unstable toward oxidation, sintering, and agglomeration. In inert media nanopowders of such size sinter at room temperature by a diffusion mechanism, and in chemically reactive media they interact with the explosion. Thus, the problem of finding the conditions of producing nanopowders that provide high dispersiveness and narrow particle size distribution within $30 \ldots 60 \mathrm{~nm}$ is topical.

\subsection{Effect of synthesis conditions on the EEW-nanop-} owders characteristics

The dispersiveness and other characteristics of nanopowders depend on all conditions of explosion - firstly, on the electrical parameters (energy consumed by wire before explosion, energy of arc stage, the velocity of energy input or power density [1-3]. The nature of wire metal and its geometry (length and diameter of wire), microstructure and substructure of wire metal, environmental properties - pressure and kind of gas, density (viscosity) also have influence on the dispersiveness and other properties of nanopowders. Besides, the properties of electroexplosive nanopowders are highly dependent on the conditions of passivation [14-16].

It is consider that the specific energy consumption of exploding wire or the ratio $e / e_{\mathrm{s}}$ is the most important parameter determining the degree of nanopowder dispersiveness and the particle size distribution function [1-3]. The specific energy consumption or the reached overheat $e / e_{\mathrm{s}}$ is a ratio of volume density of energy $e$ to energy of sublimations of wire material $e_{\mathrm{s}} ; e-$ is a ratio of the energy consumed by wire before explosion to initial volume of the wire.

The degree of dispersiveness of nanopowder can be evaluated using such characteristics as specific surface area $S_{\mathrm{sp}}$ and average surface diameter $\bar{a}_{\mathrm{s}}=6 / \mathrm{\rho} S_{\mathrm{sp}}$.

The size of particles produced by EEW decreases with

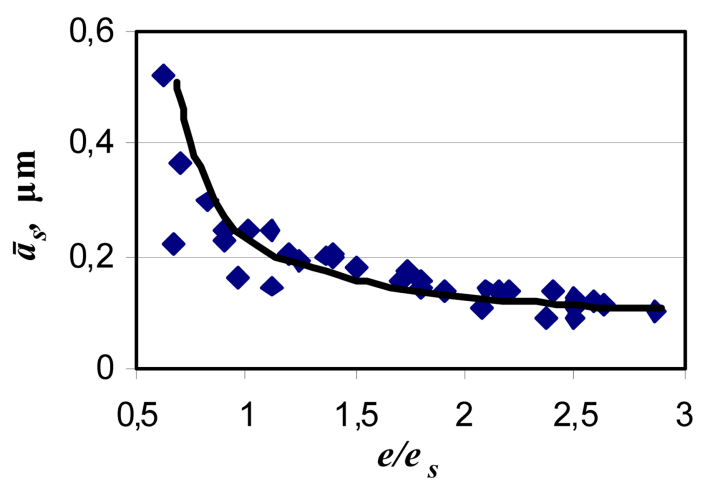

Fig. 2. Dependence of the average surface diameter of aluminum and copper particles on specific energy consumption.

increasing the the energy input in the wire (Fig. 2), and the particle size distribution becomes narrower. If the value of energy input in the wire increases from 0.8 to $(1.6 \ldots 1.8) e_{\mathrm{s}}$, the average surface diameter decreases rapidly. With further increase in the value of energy the rate of decrease of the diameter slows down, and the process of obtaining nanopowders by means this parameter becomes energetically inefficient.

The arc stage of electric discharge provides additional dispersing effect on the primary products of wires explosion $[8,9]$.

Working gas in discharge chamber at high pressure has a compression effect on explosion product and causes their sintering and coagulation. That is why the particles diameter increases (Fig. 3) if the value of gas pressure increases $[2,3,9]$.

The dispersiveness of EEW products increases with the decreasing the wire diameter $[2,3]$. However, in this case the product yield significantly decreases. It should be noted that for metal nanoparticles, the particle diameters of $30 \ldots 10 \mathrm{~nm}$ are critical ones, because smaller particles

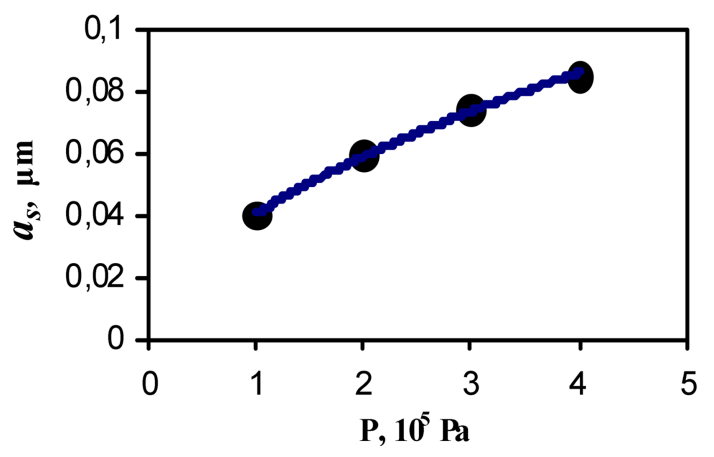

Fig. 3. Dependence of the average surface diameter of particles on the pressure (electric explosion of copper wire in argon, $\left.e / e_{\mathrm{s}}=1.2\right)$. 


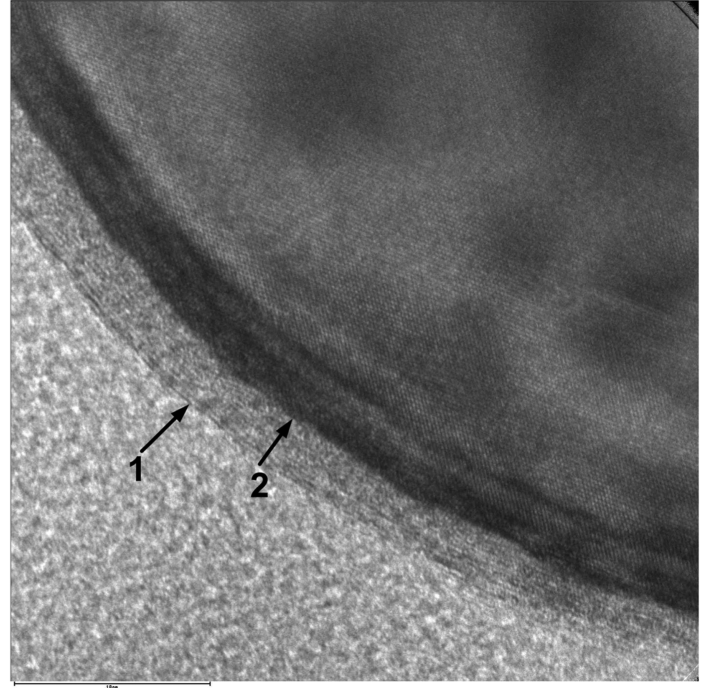

Fig. 4. TEM images of aluminum nanoparticle passivated by air: 1-oxide-hydroxide layer; 2-heavy metal impurities layer.

sinter at room and even lower temperatures.

Metal nanopowders produced by EEW in chemically inert gases have the adsorbed layer of working gas on the particles surface (Fig. 4) which does not let the particles contact and keeps them from sintering for a long period of time till the contact with air. Maximal thickness of oxide-hydroxide layer for particles with diameter $30 \ldots 50$ $\mathrm{nm}$ is not more than $7 \ldots 8 \mathrm{~nm}$, and for particles with diameter $100 \mathrm{~nm}: 3 \ldots 4 \mathrm{~nm}$. Passivation by slow oxidation in air without heating of nanoparticles enables to form the oxide-hydroxide layers. At the increase of nanopowder dispersiveness the oxides content grows up, due to that the content of unoxidized metals has threshold value depending on diameter: there is an extremely high increase of the oxidation products content at diameter less than $50 \ldots 60 \mathrm{~nm}$.

The process of passivation may be carried out just directly in the process of explosion; at that the pyrophoric property of powders reduces as well as the time required for passivation, technological process is simplified. Addition of chemically active gases $\left(\mathrm{O}_{2}\right.$ or $\left.\mathrm{N}_{2}\right)$ (5...30 vol.\%) to argon during EEW leads to the formation of passivating films on the particles and to the increase in the dispersiveness of obtained powders [7, 14]. In that case the decrease of the particles size happens because of reduction of agglomeration and sintering at the scattering and cooling the EEW products. At the same time it is observed the considerable decrease of aluminum content in nanopowder particles - up to $70 \ldots 80$

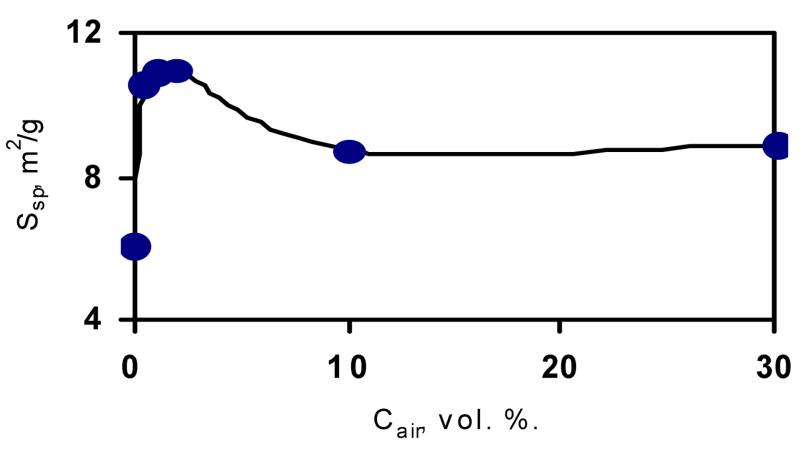

Fig. 5. Specific surface area of $\mathrm{Cu}-\mathrm{EEW}$ products in the mixture of argon and air.

wt.\%.

The effect of little addition of air $(0.5 \ldots 30$ vol. $\%)$ in argon using as working medium during electrical explosion of copper wires on dispersed, phase and chemical compositions of nanopowders was investigated [7]. Nanopowders having the highest dispersiveness were produced at the air content $1 \ldots 2$ vol. $\%: S_{\mathrm{sp}}=10.5 \ldots 11 \mathrm{~m}^{2} / \mathrm{g}$ that is corresponding to the average particle diameter $60 . .65 \mathrm{~nm}$. The dependence of the specific surface area of the obtained samples on the air content in the gas mixture is shown in Fig. 5.

The refractory compound layers of copper oxide on metal surface of particles prevent the coagulation of particles. An increase of the air content in gases mixture during EEW leads to a reduction in the metal content and to a change of the phase composition.

The output of the $\mathrm{Cu}-\mathrm{EEW}$ products in the mixture of argon and air depending on the content of air is shown in Fig. 6.

The primary disperse particles formed in the explosion particles react with oxygen in the process of recession

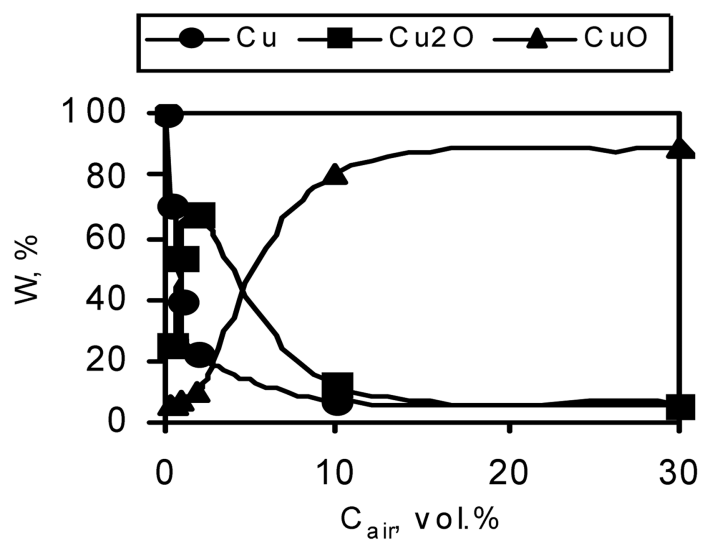

Fig. 6. The output of $\mathrm{Cu}-\mathrm{EEW}$ products in the mixture of argon and air. 
and cooling of primary EEW-products they react with air oxygen and under deficiency in oxygen and higher temperature the layer of copper oxide $\mathrm{Cu}_{2} \mathrm{O}$ forms $\left(\mathrm{T}_{\text {melt }}=\right.$ $1235^{\circ} \mathrm{C}$ ) on the particles surface. At the increase of the oxygen content in surrounding gas (in case of air content 5 vol.\% and more) $\mathrm{Cu}_{2} \mathrm{O}$ oxidizes to $\mathrm{CuO}$ in the process of cooling at lower temperature; $\mathrm{CuO}$ is less stable than $\mathrm{Cu}_{2} \mathrm{O}$ and resolves at temperature more than $1000^{\circ} \mathrm{C}$. The duration of coagulation grows up that results in lower dispersiveness of nanopowders at the increasing of air content in argon more than $10 \ldots 20$ vol. $\%: S_{\mathrm{sp}}=8.8 \mathrm{~m}^{2} / \mathrm{g}$.

The interaction of dispersed metal with working ambient during EEW can be used for synthesis of chemical compounds in nanoscale range. The density of reactants is much higher during wire explosions in condensed media compared to explosions in gases. That fact allows the chemical compounds output to be increased and their phase composition to be changed $[3,5,6,10]$.

The effect of the EEW energy parameters, density and dynamic viscosity of surrounding ambient, elemental composition of hydrocarbons $(\mathrm{C} / \mathrm{H}$ ratio in hydrocarbon molecules) on the phase compositions of nanopowders prepared by electrical explosion of tungsten, titanium and aluminium wires in liquid hydrocarbons was investigated $[3,5,6]$. Hexane $\mathrm{C}_{6} \mathrm{H}_{14}$, decane $\mathrm{C}_{10} \mathrm{H}_{22}$, benzene $\mathrm{C}_{6} \mathrm{H}_{6}$, and toluene $\mathrm{C}_{6} \mathrm{H}_{5} \mathrm{CH}_{3}$ were used as working surrounding ambient for EEW. Hydrocarbons were taken as reagents because they can give active atoms of carbon during thermodestruction.

According to the X-ray data, powders prepared by EEW in liquid hydrocarbons contain the carbides phases

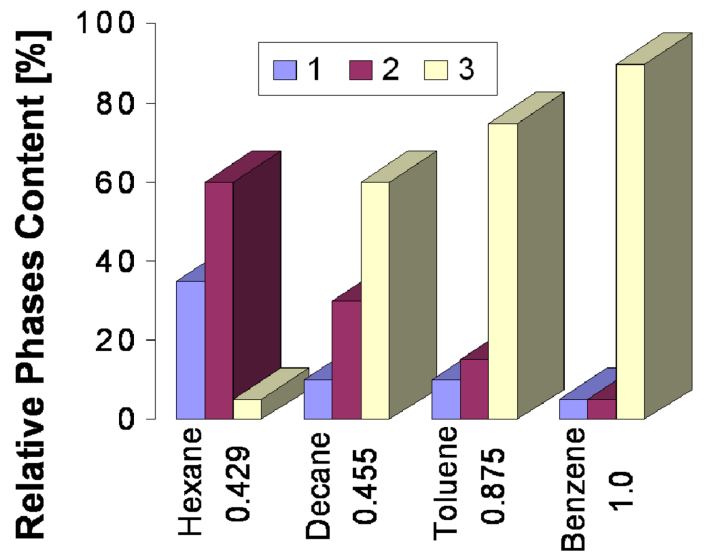

Fig. 7. Relative contents of $\mathrm{W}(1), \mathrm{W}_{2} \mathrm{C}(2)$, and $\mathrm{WC}_{1-\mathrm{x}}$ phases (3) in tungsten $\mathrm{EEW}$ products having different $\mathrm{C} / \mathrm{H}$ ratios. and residual metal. Fig. 7 shows the qualitative composition of tungsten EEW products for the indicated liquid hydrocarbon types and the energy input into the wire of $\sim 1.2 e_{\mathrm{s}}$. It can be seen from Fig. 7 that the tungsten carbide $\mathrm{WC}_{1-x}$ output increases upon increasing the $\mathrm{C} / \mathrm{H}$ ratio and the liquid hydrocarbon density, whereas the metallic tungsten content in the products decreases.

The influence of the input energy on the phase and elemental composition of EEW products was studied using decane $\mathrm{C}_{10} \mathrm{H}_{22}$ as a working ambient. According to the $\mathrm{X}$-ray data, the main product of the tungsten EEW in decane was carbide $\mathrm{WC}_{1-x}$. Even for the maximum consumed energy $\left(e / e_{\mathrm{s}}=1.2\right)$, the main phase of tungsten carbide remains the phase $\mathrm{WC}_{1-x}$ deficient in carbon.

The comparison between the formation of aluminium, tungsten, and titanium carbides produced by EEW showed that the output of the chemical compounds depends on their thermal stability. WC and TiC are more stable compounds than $\mathrm{Al}_{4} \mathrm{C}_{3}$. WC content in the explosion product is higher, $\mathrm{TiC}$ as well. The main product of aluminum EEW in decane is metal aluminum even at sufficiently high level of the input energy $\left(e / e_{\mathrm{s}}=1.5\right)$. The relative content of $\mathrm{Al}_{4} \mathrm{C}_{3}$ does not exceed $40 \%$.

Fig. 8 shows the intensities of $100 \%$-X-ray diffraction patterns of $\mathrm{Al}_{4} \mathrm{C}_{3}, \mathrm{WC}$ and $\mathrm{TiC}$ produced by $\mathrm{EEW}$ in decane.

Low output of aluminum carbide prepared by EEW can be explained by the temperature dependence of $\Delta G$ on the formation reaction of $\mathrm{Al}_{4} \mathrm{C}_{3}$. The temperature at which aluminum carbide remains stable is limited to temperatures lower than $3000 \mathrm{~K}$. During aluminum EEW in

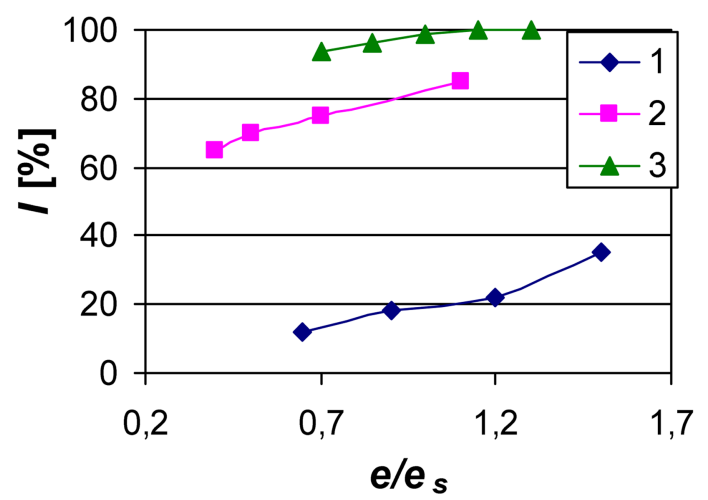

Fig. 8. Dependence of the relative contents of $\mathrm{Al}_{4} \mathrm{C}_{3}(1), \mathrm{WC}$ (2) and $\mathrm{TiC}(3)$ phases in the products of EEW in decane on the energy input in the wire. 
decane, the chemical reaction takes place due to diffusion of carbon atoms into the aluminum particle at temperatures below the boiling point of aluminum $(2720 \mathrm{~K})$ : $4 \mathrm{Al}_{\text {(liquid) }}+3 \mathrm{C}_{\text {(gas) }}=\mathrm{Al}_{4} \mathrm{C}_{3 \text { (solid). }}$ In the course of this reaction, a continuous carbide layer is formed on the metal particle surface, thereby limiting the diffusion of carbon atoms into the metal.

The maximum temperature at which $\mathrm{WC}$ and $\mathrm{TiC}$ remain stable is much higher than that for the aluminum ones. A decrease in $\Delta G$ is observed at $\sim 3000 \mathrm{~K}$. The particle morphology demonstrates the feasibility of forming tungsten carbides in the liquid phase, because the spherical particles have smooth surfaces. Hence, it follows that tungsten carbides are formed at temperatures exceeding their melting point (for example, $T_{\text {melt }} \sim 3130 \mathrm{~K}$ for $\mathrm{W}_{2} \mathrm{C}$ ) through the diffusion of carbon atoms into the liquid tungsten particle: $2 \mathrm{~W}_{\text {(liquid) }}+\mathrm{C}_{\text {(gas) }}=\mathrm{W}_{2} \mathrm{C}_{\text {(liquid). }}$. The fact that the main product of tungsten EEW in liquid hydrocarbons is nonstoichiometric tungsten carbide $\mathrm{WC}_{1-x}$ can be explained by the high concentration of active carbon atoms in the chemical reaction and by fast cooling of the prepared powders. It is known that $\mathrm{WC}_{1-x}$ remains stable at temperatures exceeding $2800 \mathrm{~K}$. Thus, the higher output of tungsten carbide nanopowders is a consequence of their higher thermal stability relative to those of $\mathrm{Al}_{4} \mathrm{C}_{3}$. The similar explanation can be given for the high output of titanium carbides in EEW.

Powders produced by electrical explosion of aluminium wires in water are different crystal modifications of aluminium oxides [2-4]. The content of oxide-hydroxides phases is defined by initial conditions of explosion and first of all, by value of the energy, input into wire. The main product formed by the electrical explosion of aluminium wire in water at specific energy less than sublimation energy is aluminium hydroxide, and at specific energy more than $e_{\mathrm{s}}$ is low-temperature modification $\gamma$ $\mathrm{Al}_{2} \mathrm{O}_{3}$. A content of residual aluminium decreases with the rise of energy consumed by wire. A drastic decrease of residual aluminium is observed in the range $e / e_{\mathrm{s}}$ $\sim 0.9 \ldots 1.1$. Formation of size composition is the result of two mechanisms: dispersion of liquid metal and condensation of vapor phase.

Peculiarity of water as an oxidation-reduction environment is the formation of lower oxides at electrical explosion of wires made of metals having multiple oxidation states, such as iron or titanium. Unlike aluminum, which is characterized by the degree of oxidation of only three, the iron can form compounds $\mathrm{Fe}^{+3}$ and $\mathrm{Fe}^{+2}$, and the titanium can form compounds $\mathrm{Ti}^{+4}$ and $\mathrm{Ti}^{+3}[3,10]$.

\subsection{The design of installation for nanopowders pro- duction}

The design of a discharge chamber also plays an important role in the formation of powders in the process of EEW. Improvement of EEW-technology was directed at reliability and productivity growth of equipment, as a result of this an industrial installation UDP-4G has been developed in High Voltage Institute at Tomsk Polytechnic University and now operates successfully. This installation was designed for producing nanopowders of pure metals and chemical compounds by EEW in gaseous working ambient. UDP-4G became widespread in many countries: South Korea, China, Germany, and United States of America. The installation UDP-4G at University of Ulsan (South Korea) is shown in Fig. 9. At the Research Centre Karlsruhe, the production of nanopowders generated during EEW was investigated at a capacitive facility DEMON-2 [17].

The scheme of installation UDP-4G is shown in Fig. 10.

Carried out investigation showed that state-of-the-art requirements to nanopowder quality specification can not be realized in powder produced at present experimentalindustrial installations like UDP-4G Not enough attention was given to the problem of the powder quality improvement.

After explosion there are multiple reflections of shock waves from the chamber sides in discharge chamber 8 of installation which result in supplementary non-adiabatic

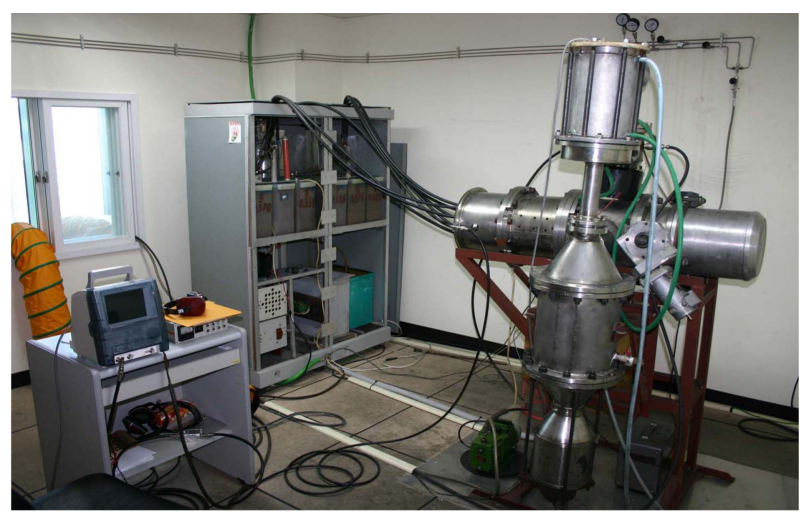

Fig. 9. Installation UDP-4G (University of Ulsan). 


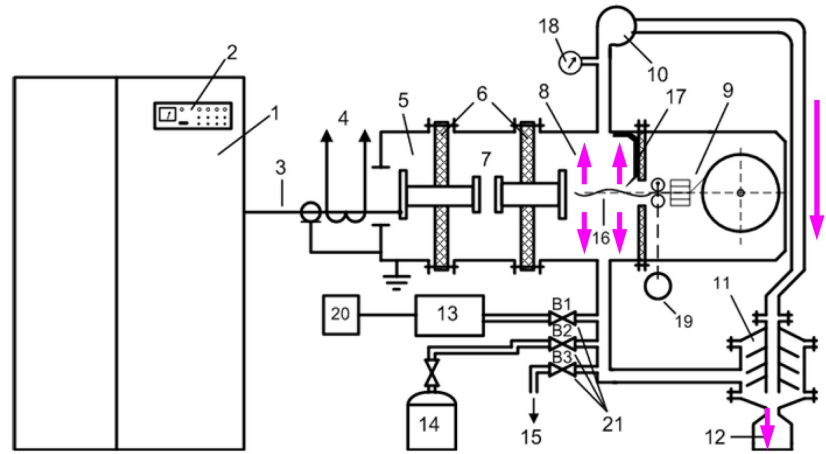

Fig. 10. Principal scheme of installation UDP-4G: 1-power supply unit; 2-control panel; 3-high-voltage cable; 4-current shunt; 5-high-voltage input chamber; 6-insulators; 7- discharge switch; 8-discharge chamber; 9-wire mechanism driving; 10ventilator; 11-filter; 12-collector of nanopowder; 13-forepump; 14-gas-cylinder; 15-exhaust ventilation pipeline; 16-exploding wire; 17-grounded electrode; 18-manometer; 19-motor; 20forepump control panel; 21-gas fitting.

compression of the explosion products and their temperature rise. This process results in thermal reflow and additional evaporation of the particles, their sintering and coagulation.

Essential disadvantage of the installation UDP-4G is compression of EEW-product flow in a tube connecting the explosive chamber and the powder collector 12. Particle number density increases during this process, possibility of agglomeration increases very much.

The used filter design at the installation UDP-4G causes that the particles of fine fraction are taken away along with gas flow before they have time to settle in powder collector. Another design defect of UDP-4G is unsuccessful location of the powder collector, which is dead end for particles on movement of shock wave after electrical explosion; as a result powder is exposed to multiple shock loads and compacts. At production of 100 gram of nanopowder the level of agglomeration is less then at production of 500 gram [18]. Upon an influence shock load the nanopowders sinter and form easily broken structure. Size of the agglomerates can reach 100 ìm and more, but after rubbing of the agglomerated powder through different meshes their sizes correspond to sizes of holes in mesh (Fig. 11).

Mentioned design disadvantages were eliminated at development of the installation UDP-5 $[12,19]$ on basis of estimation calculation, results of investigation and literature data [20]. Principal scheme of the installation

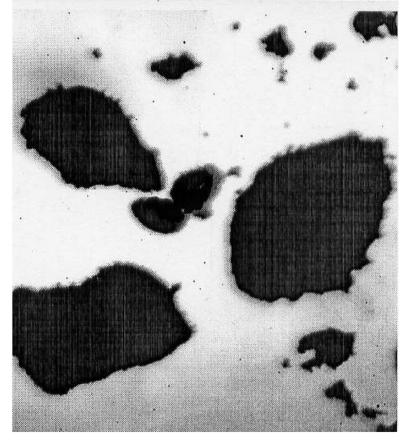

(a)

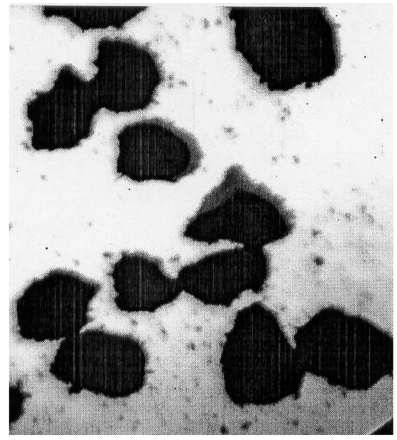

(b)
Fig. 11. Images of agglomerates after rubbing of aluminum powder through mesh $(100 \mu \mathrm{m})$ : (a) powder produced at TPU, HVRI, lab 14, (b) CETC, Tomsk (optical microscope "Biolam" x60).
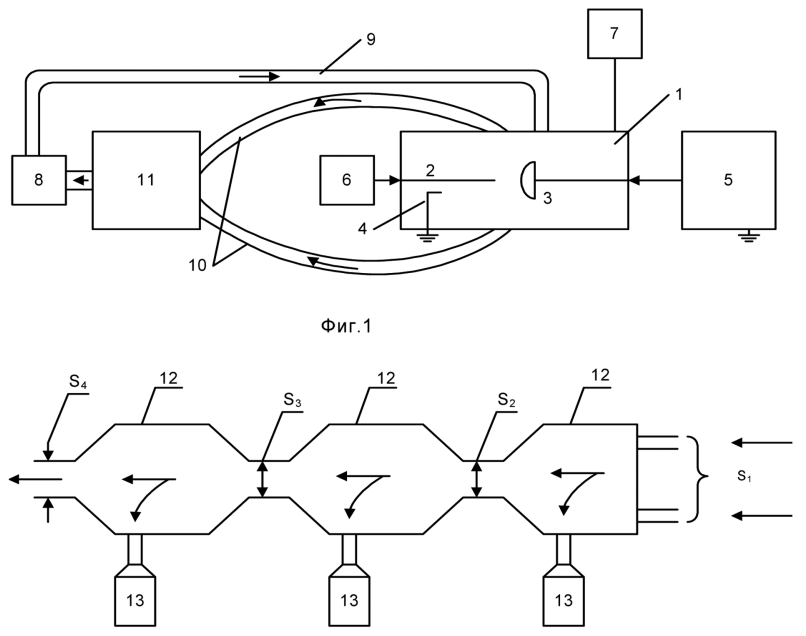

Fig. 12. Principle scheme of the installation UDP-5: 1- discharge chamber, 2-exploding wire, 3-high-voltage electrode, 4- grounded electrode, 5-power supply unit, 6-wire mechanism driving, 7 -gas filling system, 8-ventilator, 9-gas pipeline, 10-tubular gas outlets, 11-unit of gas separation and powder collector, 12expansion box, 13-powder collector.

\section{UDP-5 is shown in Fig. 12.}

The discharge chamber opposite inter-electrode gap has perforation. Attached to perforation holes in front of inter-electrode gap the special tubular gas pipes take out the explosion products into the gas separation unit and powder collector. Total flow area of perforation holes ratio to discharge chamber area according to [18] is not less than 0.6. This engineering solution allows avoiding of increasing the temperature of expanding explosion product, their vaporization, melting, and agglomeration under effect of reflected shock waves.

The long tubular pipes for transportation of EEW-products are used in UDP-5 in contrast to UDP-4G, in which 
the processes of shock waves suppression and size separation of particles happen. The tubular gas pipes between the discharge chamber and the unit of gas separation and powder collector provide more uniform distribution of EEW products in surroundings. Particles temperature decreases during their flow through long tubes and probability of particles coagulation becomes lower.

The unit of gas separation and powder collector is made in the form of the expansion boxes successively connected through the branch pipes. Each expansion box is provided with a powder collector with special size ratio [19]. Larger powder fraction separate and settle down in the process of passing through the expenders.

The comparison characteristics of nanopowders produced in the industrial installation UDP-4G and in the model of the installation UDP-5 was made in [18]. Powders were produced by electrical explosion of aluminum wires at following conditions: the wire diameter $0.3 \mathrm{~mm}$, the specific energy consumption $e / e_{\mathrm{s}}=1.6$. Size of agglomerates decreases from $60 \ldots 130 \mu \mathrm{m}$ to $2.3 \mu \mathrm{m}$, and their content in powder decreases from 60 to $6 \%$.

\section{The Application of Nanopowders and Future Research}

Our future research will focus on more detailed studying the process of EEW, on the further development of technology for production of nanopowders, on the experimental studies and selection of the most effective applications of nanopowders.

Actual problem is to find ways to stabilize the nanoparticles. The organic compounds decompose before nanopowders are beginning to oxidize, so they do not protect them from oxidation. The process of passivation of metal nanopowders by inorganic films or coatings will be studied.

The particle separation problem is not solved yet, as the problem of nanopowders production with a narrow size distribution. Our attention will be given this matter.

Study of biological effect of nanopowders in order to promote them in the technology and to develop biologically active agents is a very interesting subject.

Search for new applications of powders is also the subject of our research. Already now nanopowders find practical application in the following areas: environmental protection and remediation, catalysis, organic synthe- sis, ceramics, production of cutting tool, wear-resistant parts and coatings, lubricants and additives.

Promising areas of nanopowders application are the following:

- Energy supplements in propellants and pyrotechnics, and therefore the problem of increasing the stability of the powders with respect to oxidation appears.

- Additives in varnishes and paints, including for vehicles (to increase impact strength, wear resistance).

- Hydrogen production in mobile devices.

- Search for catalysts of burning wood, coal and turf.

\section{Conclusion}

The process of EEW as a method for nanopowders production was investigated. In pulsed fast processes such as electrical explosion of wires the formation of nanoparticles happens in strongly nonequilibrium conditions that cause some unusual properties of nanopowders. They are steady against oxidation and sintering at room temperature and characterized with high diffusion activity at the heating. The spherical form of obtained particles indicates about their stabilization through a liquid state. Very fast cooling of molten metal after wire explosion results in significant and specific crystal imperfection.

Depending on chemical composition of working ambient in the discharge chamber metal nanopowders can be produced (EEW in argon, hydrogen), metal carbides nanopowders (EEW in hydrocarbons) or metal oxides nanopowders (EEW in oxygen contained medium).

The methods for regulation of size compositions of the nanopowder produced by electrical explosion of wires are discussed: regulation of the electrical parameters (energy consumed by wire before explosion, energy of arc stage, power density); additions of chemically reactive gases into inert gas; decrease of wire diameter; properties of working media, pressure and kind of gas, design of installation.

Some directions for future research are outlined.

\section{Acknowledgments}

The authors would like to thank Research Center for Machine Parts and Materials Processing, University of Ulsan for financial support of the work. We also would 
like to thank Prof. Kwon Young-Soon for his extending interest in study of electrical explosion, providing necessary facilities and support to carry out this work.

\section{References}

[1] Y. S. Kwon, Y. H. Jung, N. A. Yavorovsky, A. P. Illyn and J. S. Kim: Scr. Mater. 44 (2001) 2247.

[2] V. Y. Ushakov, A. P. Ilyin, D. V. Tikhonov, O. B. Nazarenko and G. V. Yablunovskii. In: Proc. 7th Korea-Russian Int. Symp. Science and Technology, KORUS'2003, Vol. 1, Ulsan (2003) 175.

[3] O. B. Nazarenko, Electroexplosive nanopowders: production, properties, application, Tomsk University, Tomsk (2005). (Russian)

[4] Y. S. Kwon, O. B. Nazarenko and A. P. Ilyin: J. Kor. Powd. Met. Inst., 10 (2003) 161 (Korean).

[5] Y. S. Kwon, J. S. Kim, H. T. Kim, H. K. Lee, O. B. Nazarenko and A. P. Ilyin: J. Ind. Eng. Chem., 10 (2004) 949.

[6] Y. S. Kwon, A. P. Ilyin and O. B. Nazàrenko. In: Proc. 9th Korea-Russia Int. Symp. Science and Technology, KORUS' 2005, Novosibirsk (2005) 211.

[7] Y. S. Kwon, A. P. Ilyin, O. B. Nazarenko and D. V. Tikhonov. In: Proc. $3^{\text {rd }}$ Int. Forum Strategic Technology, IFOST 2008, Novosibirsk (2008) 214.

[8] Y. S. Kwon, A. P. Ilyin, O. B. Nazarenko, D. V. Tikhonov and G. V. Yablunovsky. In: Proc. $3^{\text {rd }}$ Int. Forum Strategic Technology, IFOST-2008, Novosibirsk (2008) 217.

[9] Y. S. Kwon, A. P. Ilyin, O. B. Nazarenko, D. V. Tikhonov and L. O. Tolbanova. In: Proc. $4^{\text {rd }}$ Int. Forum Strategic Technology, IFOST-2009, Ho Chi Minh City (2009) 132.

[10] Y. S. Kwon, A. P. Ilyin, O. B. Nazarenko, L. O. Root, D. V. Tikhonov and E. V. Startseva. In: Proc. $6^{\text {th }}$ Int. Forum
Strategic Technology, IFOST-2011, Harbin (2011) 52.

[11] L. H. Bac, K. S. Kim, J. S. Kim, J. C. Kim and C. K. Rhee: J. Kor. Powd. Met. Inst., 17 (2010) 464 (Korean).

[12] Y. S. Kwon, A. P. Ilyin, D. V. Tikhonov, O. B. Nazarenko and G. V. Yablunovskii. In: Proc. 7th Korea-Russian Int. Symp. Science and Technology, KORUS'2003, Vol. 1, Ulsan (2003) 217.

[13] Y. S .Kwon, A. P. Ilyin, D. V. Tikhonov, A. N. Grigoriev and O. B. Nazarenko. In: Proc. 20th Int. Conf. Heat Treatment, Jihlava, Czechia (2004) 419.

[14] A. P. Ilyin and A. A. Gromov, Combustion of aluminum and boron in superfine state, Tomsk State University, Tomsk (2002). (Russian)

[15] Y. S. Kwon, A. A. Gromov, A. P. Ilyin and G H. Rim: Appl. Surf. Sci., 211 (2003) 57.

[16] Y. S. Kwon, A. A. Gromov, A. P. Ilyin, A. A. Ditts, J. S. Kim, S. H. Park and M. H. Hong: Int. J. Refract. Met. Hard Mater., 22 (2004) 235.

[17] P. Hoppé, R. Böhme, W. Frey et al. In: Proc. Int. Conf. Pulsed Power Applications, Gelsenkirchen, Germany (2001) F.01/1.

[18] Y. S. Kwon, A. P. Ilyin, D. V. Tikhonov and O. B. Nazarenko. In: Proc. 8th Korea-Russian Int. Symp. Science and Technology, KORUS'2004, Vol. 1, Tomsk (2004) 227.

[19] A. P. Ilyin, O. B. Nazarenko and D. V. Tikhonov. Patent RF No 2247631.

[20] A. V. Vinogradov, V. A. Volkov, V. Y. Gidaspov and P. V. Rosovskii: Tech. Phys. Letters., 67 (1997) 19.

이 논문을 지난 30 여 년간 한국분말야금학회 발전 및 기술개발에 큰 업적을 남기신 울산대학교 권영순 교수님의 정년을 기념하여 헌정합니다. 